\title{
La recherche clinique entre industrie et médecins*
}

\section{Reto Obrist ${ }^{a}$, Jerôme Biollaz ${ }^{b}$}

a Prof. Reto Obrist, ancien médecin-directeur du Dept. Valaisan d'Oncologie

b Prof. Jerôme Biollaz, Division de Pharmacologie Clinique, CHUV, Lausanne

\footnotetext{
*et article de la série «Collaboration corps médical- industrie» reflète l'opinion personnelle de certains membres de la commission consultative et ne doit pas être comprise comme une prise de position officielle de la commission ou de l'ASSM.
}

Correspondance:

Prof. Jérôme Biollaz Division de Pharmacologie Clinique

CHUV

CH-1011 Lausanne

jerome.biollaz@chuv.ch
La recherche sponsorisée par l'industrie participe aux progrès de la médecine, à l'augmentation de nos connaissances et à la mise à disposition de nouveaux médicaments. Mais les conflits d'intérêt pour les médecins chercheurs et leurs institutions sont multiples. Les abus connus montrent les effets négatifs sur la qualité de la recherche clinique et ses conséquences pour les patients et les ressources des systèmes de santé et - le point peut-être le plus dramatique - pour l'intégrité éthique et la crédibilité de toute la recherche clinique.

Cet article souhaite montrer comment on pourrait y remédier (du moins partiellement) en suivant les directives de l'Académie Suisse des Sciences Médicales (ASSM) [1] et en illustrant quelques points pertinents par des références à des études scientifiques. Si cet article s'intéresse plus particulièrement aux relations avec les industriels du médicament, la majorité des points soulevés s'applique également aux autres industries de la santé (medical devices, en particulier).

\section{Évidences}

Les évidences d'une influence majeure de l'industrie pharmaceutique sur la recherche clinique sont abondantes. Des prises de position de sociétés et d'autorités américaines et britanniques soulignent, qu'il s'agit d'un problème général, qui remet en cause la crédibilité de la recherche clinique [2-5].

Les études cliniques sont sponsorisées par l'industrie à quelque $80 \%$. Ces études présentent souvent des biais en faveur de la substance du sponsor comme démontré par plusieurs métaanalyses [6-8]. Employés du sponsor comme coauteurs, mais plus souvent encore contributeurs clandestins n'apparaissant pas sur la publication $[9,10]$ sont une partie du problème. Les résultats se trouvent particulièrement «influençables» dans les études pharmaco-économiques, utilisées pour justifier l'introduction ou le prix d'une substance [11]. La conduite d'une étude peut être influencée par des modifications, parfois subtiles, en faveur de la substance testée, voir en défaveur du comparatif, soit concernant son efficacité ou les effets secondaires [12].

Les méta-analyses conduites et financées sans soutien industriel sont, en comparaison directe, de qualité supérieure à celles avec un tel soutien et présentent moins de biais en faveur de la substance étudiée. Ainsi la substance du sponsor est favorisée dans $40 \%$ des études industrielles contre $20 \%$ dans les autres $[13,14]$. Les méthodes utilisées pour obtenir ces résultats favo- rables sont parfois décelables en comparant le protocole de l'étude avec la publication finale. C'est ainsi, par exemple, que dans 11 paires sur 62 analysées, les données étaient systématiquement identiques, alors que dans les autres elles étaient souvent modifiées en faveur de la substance du sponsor [15-17].

Des résumés de congrès rapportent souvent des risques relatifs et des rapports de cote (odds-ratios) significatifs, alors qu'en réalité ces calculs très préliminaires sont souvent erronés. De tels résultats ne devraient pas être acceptés sans revue de la publication intégrale [18]. Des présentations sélectives de résultats favorables, voire même des conclusions fausses, se glissent parfois dans les dossiers soumis aux autorités de réglementation pour approbation [19]. Finalement les biais de publication représentent un problème majeur pour qui veut pratiquer une médecine fondée sur les évidences (EBM). Ainsi, en Suisse, le $52 \%$ seulement des études approuvées par un comité d'éthique Bernois a été finalement publié. On ignore les raisons de la non-publication de la moitié des études, dont la majorité sponsorisée par l'industrie [20].

\section{Les directives}

1. Les essais cliniques sont conduits selon les principes des «good clinical practice» (GCP).

Le but des GCP est - sur la base des principes de la déclaration d'Helsinki - d'abord la protection des sujets participant à une étude clinique (droits, sécurité, bien-être) et ensuite d'assurer la crédibilité des résultats [21]. Les directives GCP mettent des jalons obligatoires pour les chercheurs, les comités d'éthique et les industriels du médicament. La loi suisse sur les produits thérapeutiques (LPTh) y fait référence. Elles ont également influencé la loi sur la recherche humaine, actuellement en discussion. La surveillance des projets de recherche incombe aux comités d'éthique et à Swissmédic, qui s'investissent surtout dans l'analyse initiale des protocoles soumis, nettement moins, voire pas du tout, dans l'exécution des études et leur mise en valeur (rapport final, publication des résultats).

\section{Les institutions pratiquant la recherche clinique évaluent régulièrement la qualité de cette recherche.}

Cette évaluation est complexe et difficile. Comme discuté ci-dessus, la littérature montre des biais mul- 
tiples et systématiques dans la réalisation des études cliniques. Une telle évaluation demande non seulement une grande expertise mais aussi des ressources majeures, allant probablement bien au delà des possibilités de beaucoup d'institutions.

L'«Institute of Medicine» des académies américaines propose pour cette raison non seulement une surveillance de la qualité, mais l'établissement de commissions institutionnelles pour la gestion des conflits d'intérêt. Les institutions dans lesquelles se pratiquent des études cliniques (facultés/hôpitaux) se doivent d'établir des directives pour la gestion de ces conflits et, surtout, de veiller à leur application. Ces actions devraient être activement promues par les instances de surveillance [2].

\section{Tous les essais cliniques sont répertoriés dans un registre central.}

Cette mesure est également stipulée dans la loi sur la recherche humaine en discussion. Un des objectifs poursuivis est la détection de la nonpublication d'études, donc de la publication sélective de résultats favorables («publication bias») [22]. Cette mesure a également été prise par les grandes revues scientifiques, qui demandent, avant toute considération pour publication, un enregistrement préalable dans une base de données (par ex. <clinicaltrials.gov>) de toute étude clinique. Vu les «divergences» trop fréquemment constatées entre le protocole d'étude et la publication, la simple notification de l'étude est probablement insuffisante et seule une publication de tous les éléments clefs du protocole dans une telle base de données paraît à même d'atteindre les objectifs poursuivis.

\section{L'investigateur responsable et ses collaborateurs ne sont liés à l'essai ou à son résultat par aucun intérêt d'ordre financier.}

Ce point ne peut être validé que s'il y a transparence complète des relations financières et si ses relations sont réglées contractuellement (voir le point suivant). Indépendamment de l'importance attribuée par les patients à cette transparence, objet d'un récent éditorial du New England Journal of Medicine, elle semble indispensable au maintien de l'intégrité éthique de la recherche et de sa perception dans le public [23].

\section{Le déroulement et le financement des essais sont régis contractuellement.}

Ce point et le suivant préconisent une définition claire de la relation entre sponsor et investigateur. Sont concernés surtout les flux financiers, qui doivent être séparés de l'intérêt personnel de l'investigateur. La motivation financière de l'institution est un problème éthique qui n'a pas été sérieusement abordé à ce jour. Le contrat doit être porté à la connaissance du comité d'éthique et doit comporter non seulement les aspects financiers mais tous ceux liés à l'exécution de la recherche, afin de pouvoir y déceler et s'opposer aux clauses «bâillons» («gag-clauses») qui ont trop souvent permis de séquestrer les données défavorables au produit étudié.

\section{Les prestations financières fournies dans le cadre d'un essai doivent être versées sur des comptes tiers institutionnels.}

En somme: pas de caisses noires et pas de profit individuel, mais des rémunérations adéquates pour les prestations fournies.

\section{Le déroulement d'essais cliniques et l'achat de produits du promoteur sont indépendants l'un de l'autre.}

Les médecins impliqués dans des études cliniques sont généralement ceux qui connaissent le mieux la substance, avec parfois des années d'expériences avant son enregistrement par Swissmédic. Ils sont pour cette raison consultés par les commissions institutionnelles des médicaments. Leur expertise indéniable est très utile, mais elle ne saurait, seule, dicter la politique institutionnelle du médicament. Ces investigateurs pourront donner leur avis mais se garderont de vouloir imposer leur point de vue.

\section{Lors de la publication et la présentation} des résultats d'un essai, le financement de celui-ci doit être divulgué.

La grande majorité des revues scientifiques exigent cette déclaration. Mais pour que ces déclarations aient une quelconque valeur, elles devraient figurer dans des bases de données facilement accessibles et gratuites. Les éditeurs de revues médicales devraient prendre des mesures adéquates en cas de manquement à cette obligation.

9. L'interprétation des résultats d'un essai doit être indépendante des intérêts du promoteur.

Les intérêts du sponsor sont omniprésents dans la recherche clinique, leur contrôle s'avère donc très difficile. La seule mesure efficace serait un financement neutre, indépendant de l'industrie, pour toute étude jugée essentielle pour des raisons scientifiques ou de santé publique. Le déséquilibre de la balance pourrait être quelque peu redressé et les biais minimisés. Actuellement, les fonds nécessaires au financement de telles études sont inexistants en Suisse.

10. Les chercheurs ne collaborent pas aux activités de marketing en faveur de produits ayant fait l'objet d'essais auxquels ils ont participé.

Si la participation des investigateurs à des « advisory boards » peut se justifier, lorsque ce nom ne camoufle pas la participation à des activités de marketing, des invitations ou citations comme «opinion leader» sont problématiques puisque, de l'aveu même des industriels du médicament, l'objectif est clairement commercial [24]. 
La corruption de la formation (colloques de formation continue «orientés», articles signés par des auteurs prête-nom, directives thérapeutiques rédigées «sous influence») est une réalité.

\section{Conclusion}

Cet article vise à sensibiliser le corps médical aux influences de l'industrie pharmaceutique sur les résultats de la recherche clinique. Il incombe aux chercheurs de veiller à l'intégrité de la recherche clinique et aux universités et hôpitaux de réglementer cet aspect afin d'éviter la mise en place, sous la pression politique, d'une législation encore plus contraignante, alourdissant la charge financière déjà extrême de la recherche clinique. Les multiples scandales publiés régulièrement dans les médias professionnels et grand public et les analyses scientifiques du comportement des chercheurs doivent sensibiliser les «consommateurs» des résultats des études cliniques: médecins praticiens, enseignants des facultés et de formation continue, public et médias. L'utilisation à visée commerciale des résultats des études cliniques va se poursuivre. Il nous appartient d'analyser de manière critique ces résultats et leur présentation pour en connaître leur vraie valeur scientifique. Les directives de l'académie sont à cet égard un instrument utile.

\section{Références}

1 ASSM. Directives «Collaboration corps médicalindustrie». Available at: http://www.samw.ch/fr/ Ethique/Corps-medical-Industrie.html.

2 Steinbrook R. Controlling conflict of interest proposals from the Institute of Medicine. N Engl J Med. 2009;360(21):2160-2163.

3 House of Commons. House of Commons Health Committee. The influence of the pharmaceutical industry. Available at: www.publications.parliament. uk/pa/cm200405/cmselect/.../42.pdf.

4 Ferner RE. The influence of big pharma. BMJ. 2005;330(7496):855-856.

5 Rothman DJ, McDonald WJ, Berkowitz CD, u. a. Professional medical associations and their relationships with industry: a proposal for controlling conflict of interest. JAMA. 2009;301(13):1367-1372.

6 Winther FO, Hole OP, Nitter-Hauge S. An analysis of the clinical development of drugs in Norway for the years 2000 and 2004: the influence of the pharmaceutical industry. Eur J Clin Pharmacol. 2007;63(10):909-12.

7 Montgomery JH, Byerly M, Carmody T, u. a. An analysis of the effect of funding source in randomized clinical trials of second generation antipsychotics for the treatment of schizophrenia. Control Clin Trials. 2004;25(6):598-612.

8 Als-Nielsen B, Chen W, Gluud C, Kjaergard LL. Association of funding and conclusions in randomized drug trials: a reflection of treatment effect or adverse events? JAMA. 2003;290(7):921-928.
9 Buchkowsky SS, Jewesson PJ. Industry sponsorship and authorship of clinical trials over 20 years. Ann Pharmacother. 2004;38(4):579-85.

10 Gøtzsche PC, Hróbjartsson A, Johansen HK, u. a. Ghost authorship in industry-initiated randomised trials. PLoS Med. 2007;4(1):e19.

11 Baker CB, Johnsrud MT, Crismon ML, Rosenheck RA, Woods SW. Quantitative analysis of sponsorship bias in economic studies of antidepressants. Br J Psychiatry. 2003;183:498-506.

12 Jørgensen KJ, Gøtzsche PC, Johansen HK. Voriconazole versus amphotericin B in cancer patients with neutropenia. Cochrane Database Syst Rev. 2006;(1):CD004707.

13 Jørgensen AW, Maric KL, Tendal B, Faurschou A, Gøtzsche PC. Industry-supported meta-analyses compared with meta-analyses with non-profit or no support: differences in methodological quality and conclusions. BMC Med Res Methodol. 2008;8:60.

14 Jørgensen AW, Hilden J, Gøtzsche PC. Cochrane reviews compared with industry supported metaanalyses and other meta-analyses of the same drugs: systematic review. BMJ. 2006;333(7572):782.

15 Chan A, Hróbjartsson A, Jørgensen KJ, Gøtzsche PC, Altman DG. Discrepancies in sample size calculations and data analyses reported in randomised trials: comparison of publications with protocols. BMJ. 2008;337:a2299.

16 Pildal J, Hróbjartsson A, Jørgensen KJ, u. a. Impact of allocation concealment on conclusions drawn from meta-analyses of randomized trials. Int J Epidemiol. 2007;36(4):847-57.

17 Pildal J, Chan A, Hróbjartsson A, u. a. Comparison of descriptions of allocation concealment in trial protocols and the published reports: cohort study. BMJ. 2005;330(7499):1049.

18 Gøtzsche PC. Believability of relative risks and odds ratios in abstracts: cross sectional study. BMJ. 2006;333(7561):231-234

19 Svensson S, Mansfield PR. Escitalopram: superior to citalopram or a chiral chimera? Psychother Psychosom. 73(1):10-6.

20 von Elm E, Röllin A, Blümle A, u. a. Publication and non-publication of clinical trials: longitudinal study of applications submitted to a research ethics committee. 2008;138(13-14):197-203.

21 EMEA (CPMP/ICH/135/95). Guideline for Good Clinical Practice.ICH Harmonized Tripartite Guideline.

22 Mathieu S, Boutron I, Moher D, Altman DG, Ravaud P. Comparison of Registered and Published Primary Outcomes in Randomized Controlled Trials. JAMA. 2009;302(9):977-984.

23 Weinfurt K.P. NEJM - Disclosure of Financial Relationships to Participants in Clinical Research. 2009. Available at: http://content.nejm.org/cgi/ content/full/361/9/916.

24 Moynihan R. Key opinion leaders: independent experts or drug representatives in disguise? BMJ. 2008;336(7658):1402-1403. 